\title{
Anaphylaxis to Moringa oleifera: First description
}

\author{
Lucinda J. Berglund ${ }^{1,2}$
}

1. Department of Immunopathology, NSW Health Pathology, ICPMR Westmead Hospital, Australia

2. Faculty of Medicine, University of Sydney, Australia

\section{CASE STUDY}

Please cite this paper as: Berglund LJ. Anaphylaxis to Moringa oleifera: First description. AMJ 2018;11(3):176177. https://doi.org/10.21767/AMJ.2018.3344

Corresponding Author:

Dr Lucinda J. Berglund

Department of Immunopathology, NSW Health Pathology, ICPMR, Westmead Hospital, Australia

Email: Lucinda.Berglund@health.nsw.gov.au

\section{ABSTRACT}

We describe the first reported case of repeated anaphylaxis after ingestion of Moringa oleifera, causing significant hypotension, angioedema and elevation of serum tryptase. Moringa oleifera seedpod was confirmed as the causative allergen by skin testing with the fresh pod. Moringa oleifera is widely consumed, both as a vegetable and in herbal medicines.

\section{Key Words}

Moringa oleifera, anaphylaxis, angioedema

\section{Implications for Practice:}

\section{What is known about this subject?}

Moringa oleifera has been described as having anti-allergic potential, and is frequently used as a herbal remedy for a range of conditions.

\section{What new information is offered in this case study?}

This is the first description of systemic anaphylaxis following ingestion of Moringa oleifera seedpod.

3. What are the implications for research, policy, or practice?

Even commonly eaten vegetables can have anaphylactic potential, and this should be considered when Moringa oleifera is ingested as a herbal remedy.

\section{Background}

The Moringa oleifera plant, also known as the Drumstick tree, is widely consumed in India and neighbouring countries, both as a vegetable and in traditional medicine for various conditions, including allergies, inflammation and cancer. ${ }^{1}$ The leaves, stem and seedpod of Moringa oleifera can all be eaten, and contain antioxidant compounds and a range of nutrients. ${ }^{1}$ We describe the first reported case of anaphylaxis after ingestion of the young seedpod of $M$. oleifera. As anaphylaxis is a potentially life-threatening condition, individuals choosing to consume $M$. oleifera as a herbal remedy should be aware of its anaphylactic potential. After any episode of anaphylaxis, it is important to identify the causative allergen in order to prevent recurrent anaphylaxis on further exposure. Allergen-specific IgE can be identified through serum IgE testing or skin prick testing. This case illustrates the role of skin prick testing with a fresh plant to demonstrate IgE-sensitisation.

\section{Case details}

A 40 year old Australian office-worker of Indian heritage developed widespread angioedema over his face and body within 20 minutes of ingesting cooked $M$. oleifera, the young seedpod of the Drumstick tree. He had frequently eaten this vegetable in this past without symptoms. On this occasion, he presented to the Westmead Hospital Emergency Department in Sydney, Australia, hypotensive and tachypnoeic, with a blood pressure of $85 / 51 \mathrm{mmHg}$. He responded rapidly to intramuscular adrenaline and parenteral glucocorticoids and antihistamines. A serum tryptase shortly after admission was elevated at 39.6ug/L (reference range $0-11.4 \mathrm{ug} / \mathrm{L}$ ), consistent with anaphylaxis. A month later, his tryptase had returned to normal at $7.0 \mathrm{ug} / \mathrm{L}$.

He avoided eating $M$. oleifera again until four months later, when he ingested a potato curry cooked with $M$. oleifera pod. Within ten minutes, he developed marked facial angioedema, lower limb pruritus and hypotension, with a blood pressure of $90 / 60 \mathrm{mmHg}$, which again responded to 
adrenaline, glucocorticoids and antihistamines. A serum tryptase was elevated to $30.8 \mathrm{ug} / \mathrm{L}$.

Skin prick testing to the pulp of a fresh $M$. oleifera pod containing seeds was positive with a $4 \mathrm{~mm}$ wheal, with a positive histamine control and negative saline control. Prickto-prick testing through the skin only of the M. oleifera pod was negative. A health care worker, who had never experienced symptoms after $M$. oleifera ingestion, was selected as a healthy control, in order to ensure that the patient's positive wheal was not caused by non-specific skin irritation rather than IgE-mediated histamine release. Skin prick testing to both the pulp and the skin in the healthy control individual were negative, and a positive histamine control and negative saline control were also demonstrated in this individual.

\section{Discussion}

Both the seeds and leaves of $M$. oleifera have been described as possessing properties countering allergy and inflammation. M. oleifera seed extracts have been reported to inhibit histamine release from mast cells and prevent anaphylaxis, ${ }^{2}$ and reduce eosinophil counts and airway inflammation in animal models of asthma. ${ }^{3,4}$ Topical application of $M$. oleifera leaf extracts are reported to reduce inflammatory cytokines in atopic dermatitis. ${ }^{5}$

Conversely, occupational asthma induced by $M$. oleifera seed powder has been described, ${ }^{6}$ with positive skin prick testing and reproducible symptoms on challenge. To our knowledge, there are no reports of severe anaphylaxis with hypotension and an elevated tryptase after $M$. oleifera ingestion, as has occurred in this patient. Based on his skin prick testing, which demonstrated histamine release in response to the pulp containing seeds but not to the skin, it seems likely that this patient developed IgE sensitisation to M. oleifera seeds. It is possible that the seeds contain different allergens that are not present in the leaves. Differentiating the allergenic potential of seeds versus leaves may be important in individuals who develop allergic symptoms after ingesting $M$. oleifera seed extracts as herbal therapy.

\section{Conclusion}

This case presents a reminder that although herbal therapies may often appeal to patients due to their natural, plant-based origins, and may contain active components with therapeutic efficacy; these plants are also capable of inducing aberrant allergic responses, which can be potentially life-threatening. Skin prick testing to fresh food can be a useful diagnostic tool, as long as adequate positive and negative controls and unaffected healthy controls are in place. Despite reports of $M$. oleifera reducing histamine release from mast cells, a positive skin prick test to fresh $M$. oleifera seedpod can still be demonstrated in the context of IgE sensitisation and anaphylaxis.

\section{References}

1. Abdull Razis AF, Ibrahim, MD, Kntayya, SB. Health benefits of Moringa oleifera. Asian Pac J Cancer Prev. 2014;15(20):8571-8576. doi: 10.7314/APJCP.2014.15.20.8571.

2. Mahajan SG, Mehta AA. Inhibitory Action of Ethanolic Extract of Seeds of Moringa oleifera Lam. On Systemic and Local Anaphylaxis. J Immunotoxicol. 2007;4:287294. DOI: 10.1080/15476910701680137.

3. Goyal BR, Goyal RK, Mehta AA. Investigation into the mechanism of anti-asthmatic action of Moringa oleifera. J Diet Suppl. 2009;6(4):313-327. doi: 10.3109/19390210903280199.

4. Mahajan SG, Mehta AA. Suppression of ovalbumininduced Th2-driven airway inflammation by $\beta$-sitosterol in a guinea pig model of asthma. Eur J Pharmacol. 2011;650(1):458-464. doi: 10.1016/j.ejphar.2010.09.075.

5. Choi EJ, Debnath $\mathrm{T}$, Tang $\mathrm{Y}$, et al. Topical application of Moringa oleifera leaf extract ameliorates experimentally induced atopic dermatitis by the regulation of Th1/Th2/Th17 balance. Biomed Pharmacother. 2016;84:870-877. doi: 10.1016/j.biopha.2016.09.085.

6. Poussel M, Penven E, Richard C, et al. Occupational asthma to "the miracle tree" ( Moringa oleifera ): first description. Journal of Allergy and Clinical Immunology In Practice. 2015;3(5):813-814. doi: 10.1016/j.jaip.2015.05.016.

\section{PEER REVIEW}

Not commissioned. Externally peer reviewed.

\section{FUNDING}

None

\section{PATIENT CONSENT}

The author, Berglund $L J$, declares that:

1. They have obtained written, informed consent for the publication of the details relating to the patient(s) in this report.

2. All possible steps have been taken to safeguard the identity of the patient(s).

3. This submission is compliant with the requirements of local research ethics committees. 